\title{
Diagnostic Imaging Bull's Eye
}

National Cancer Institute

\section{Source}

National Cancer Institute. Diagnostic Imaging Bull's Eye. NCI Thesaurus. Code C94883.

A color scale or grayscale display of polar maps as concentric annuli. 